\title{
Software Application for Remote Monitoring of Fleets Based on Geographic Information Systems Using Open Source Technologies.
}

\author{
http://dx.doi.org/10.3991/ijoe.v8iS3.2251 \\ J.D. Cano, M.J. Remedios and P.E. Palau \\ University of Computer Sciences, La Habana, Cuba
}

\begin{abstract}
Controlling a fleet usually implies to establish means of control of vehicles, to collect the data associated with the routes taken by these vehicles, to interpret and evaluate the meaning of the collected data and to make the appropriate decisions to improve the efficiency in the use of vehicles in an organization. The implementation of this process of fleet management is mainly performed manually and the solutions available on the market are costly because of the payments for licenses, it is also necessary that the people monitoring the fleets are geographically close to them. This paper aims to answer the following questions: How to reduce errors in the management of information resulting from the fleet management process? How to reduce the cost of remote fleet monitoring? To obtain the solution, we propose the use of GPS devices in each vehicle, the GPS device's information is captured and consistently stored in a data base, then the information is consulted, analyzed and represented on a map. The result is a software application that allows users have fast and reliable information that will enable them to take the necessary decisions in the vehicle fleet they are trying to control at a low cost.
\end{abstract}

Index Terms-Remote monitoring, fleet management, geographic information systems, open source.

\section{INTRODUCTION}

Almost every organization in today's world depends on a fleet of vehicles to implement activities relating to the duties they perform. In many cases these fleets can have a large number of vehicles which makes the measurement of the efficiency with which these fleets operate and the decisions taking that helps to improve this efficiency an important issue. Controlling a fleet usually implies to establish means to control vehicles, defining in each case the means to use in order to have effective control over the fleet, to define the mechanisms to collect the data associated with the routes of the vehicles during the working day, to interpret the collected data, assessing the data to allow the decisions taking process that helps improve the efficiency in the use of vehicles in the organization. The approach an organization chooses or is forced to use to exercise control over their vehicle fleet has a direct impact on the efficiency with which the fleet operates as the use of either approach influences on several variables inherent to the operation of the fleet, for example, the reliability of the information available to make decisions regarding the management of the organization's fleet, the planning of the routes to be followed by vehicles, the discipline of the drivers related to the strict compliance of the planned route, the time with which deficiencies in the manner in which the fleet operates are identified and others such as the cost of controlling the fleet, and so on. This paper aims to answer the following questions: How to reduce errors in the management of information resulting from the fleet management process? How to reduce the cost of controlling a fleet at a distance? In response to these questions, finally, we propose to obtain a software solution based in the use of GPS devices in each vehicle and then we seek to capture device information, store it consistently and then consult it, analyze it and represent it on a map. The result is a software application that allows users anywhere in the world, have fast and reliable information that will enable them to take the necessary decisions regarding the operation of the vehicle fleet at a cost equivalent only to the purchase of GPS devices plus the hiring of a hosting service on the Internet.

\section{FLEET MANAGEMENT}

Fleet management is the mechanism to check and verify the behavior of a fleet of vehicles according to indicators such as the location, speed, routes and paths traveled. It's performed in order to achieve the objectives that were defined during the planning of these resources, also to detect any deviation from normal patterns previously established and correct these deviations in the behavior of the fleet. Clearly there are several ways to accomplish the foregoing, the approach used to do this is always the prerogative of the organization, but the decision is usually influenced by several factors including: the economic constraints of the organization when purchasing technology components that helps to exercise effective control over the fleet.

\section{A. Approaches.}

The approaches applied by organizations in the world vary depending on the characteristics of the situation in which each particular organization operates. For example:

Some organizations with mayor economic limitations choose assign rout forms to drivers, then the drivers should write on the form every move they make in the vehicle and at the end of a time interval that is defined by the organization, these route forms are collected and analyzed to make decisions about the performance of the fleet and its drivers in the defined time interval, this way of proceeding has, a priori, several disadvantages: 
1. The information resulting from the control process is managed manually by the executers of the process, leading to the generation of several errors and inconsistencies in the information.

2. It creates a whole bureaucratic mechanism that causes slowness in detecting deficiencies regarding to the way in which the fleet operates and therefore creates an unnecessary delay in correcting these problems.

3. It leaves room for fraudulent conduct because the vehicle activity report is generated by the person driving the vehicle and, after all, is the person that is being monitored.

4. The number of people involved in the control process it's significantly increased because it will include: the drivers in charge of updating the route forms, routes inspectors in charge of detecting drivers who travel without updating the route forms, specialists in charge of collecting and analyzing the information generated in a time interval and finally the executives in charge of taking the decisions regarding to the operation of the fleet.

On the other hand, many organizations have preferred the use of information and communications technologies (ICT) during the process of fleet management, the actions of these organizations relies heavily on the "Fleet Tracking" concept which is defined as the use of GPS devices to identify, locate and obtain reports of one or more vehicles. The rapid and reliable access to the position history of each vehicle of a fleet allows increased responsiveness to changing travel conditions. In 2008, a study entitled "Improving Productivity and Profitability Through Service Fleet Management" [1] published by the "Aberdeen Group" describes how fleet management, using this approach, affects large and small organizations, the report said that many organizations choose this approach to manage their fleets because they can achieve:

- Reduced response times for services (57\%)

- Reduced operating costs related to the fleet (46\%)

- Increased productivity related to services (41\%)

- Extended the life of the vehicle (16\%)

There are several solutions in the global software market that allow any organization to implement this approach. For example: "Localización GPS Vehículos" from the Spanish company MICRONAV, "Localización de Flotas" from the Mexican company GLOBALSAT, "OODISMAL" from the advanced information systems group than belongs to the University of Zaragoza, "Fleet Control" from the American company CES WIRELESS TECHNOLOGIES CORP. and these are just some of the solutions that can be found. Although it is just to say that all of them offer an efficient solution in applying ICT to fleet control, among the major disadvantages of their use are, first: they are proprietary software products and therefore do not share their design nor their source code, making it impossible to add other features, to the software solution, that might be required from a particular organization. Both "GlobalSat Localización de Flotas" as "Micronav Localización GPS Vehículos", "OODISMAL" and "Fleet Control" are software solutions that require permission trough licenses from their owners for exploitation, this implies an additional cost for the acquisition of the software solution, a luxury that not every organization can afford.

\section{GEOGRAPHIC INFORMATION SYSTEM FOR FLEET MANAGEMENT}

Geographic Information Systems (GIS) are part of the most extensive environment of the Information Systems, being able to integrate, to store, to publish, to analyze, to share and to show the information geographically indexed.

The space data are the fundamental component of each GIS application. Specifically we may say that the space data are variables associated to localization in the space, the forms of a geographical object and the relationships among them, commonly with coordinates and topologies [2]. The representation of these data through maps and symbols that represent the geography like geometric forms is denominated geographical information and it is considered that $80 \%$ of the existent corporate data in the entire world have this component [3].

According to José Manuel Miñano [4], other GIS's main components that guarantee their operation are the packages that contain the tools and methods that are required to generate, to store, to analyze and to deploy the geographical information. Another fundamental component are the devices where the system will operate, plus the optional peripherals that have their utility mainly in the input and output of data, like the GPS devices. GPS devices allow to determine, with high precision, the position of an object on the entire world because they work with direct data sources and the data generation takes place recording the position on the most fundamental entity available, the earth itself. Using GPS devices turns out to be more advantageous than using a map or a picture of a part of the terrestrial surface that doesn't includes the constant transformation process of the object. That's why the most efficient and precise form of using a GPS device would be to develop a database and to pick up the space data and the object's attributes data at the same time. Some GPS receivers allow this type of data collection, and these data in turn can be the information input to a GIS. The use as a whole of these two technological developments (GPS plus GIS) has become a powerful alternative to develop software solutions that bring agility and speed to the information management process. In this context the "Geographic Information System for Fleet Management” project is created.

There is no doubt that using the "Fleet Tracking" approach offers very tentative advantages for any organization. However, the cost of acquiring a software solution can be a problem, mainly for the payments of licenses to the solution's developers. At the University of Computer Sciences (UCI) an application called "Geographic Information System for Fleet Management (SIGFlotas)" is being developed and several releases have been made during the production process. SIGFlotas has been developed in order to answer both questions posed on the introduction of this paper.

\section{A. Methods and Tools}

In order to answer the question: How to reduce the cost of controlling a fleet at a distance? We conducted a survey of tools for software development. The result of the study was a selection of "Open Source" tools under the GNU GPL license completely. 
To develop the user interface we selected the EXTJS library, EXTJS is a library developed in "javascript", it's generally used in systems requiring high levels of user interaction, communication with the server is performed in background, allowing requesting or sending data and processing the information received in real time. To work with the maps we selected OpenLayers javascript library, OpenLayers is a free and "Open Source" software project that handles the displaying of geographic information and allows manipulation of the map through basic navigation and analysis tools. To perform server-side information management we selected PHP language because it is distributed for free under an open license, PHP is a language simple and comfortable, also it has as most relevant features its speed, it's multi-platform functioning, being able to run on various operating systems, diverse web servers and databases. Finally, we selected PostgreSQL as database management system to ensure the persistence of system's information, say the data of the vehicles, drivers, planned routes and information collected from GPS devices.

The tools selection made possible to eliminate payments for licenses completely and also made possible to obtain a software solution entirely based on open source software which solves the problem of adding future functionality without having to engage the services of the original developers to do so.

\section{B. Funcional Characteristics.}

Having selected a set of software development tools that eliminates the costs of license fees, the study focused on answering the question: How to reduce errors in information management resulting from the process of controlling a fleet at a distance?, the solution was to execute a requirements engineering process that allowed us to identify the functional characteristics to be developed, to automate the process of controlling a fleet. From a study of the problem domain the following functional requirements $(\mathrm{RF})$ were identified:

The system must:

RF1. Allow the user to identify itself in order to assign the permissions inherent to its role.

RF2. Allow the system administrator to manage the information of the system's users.

2.1 Allow adding the data associated to a system's user. 2.2 Allow modifying the data values of a system's user. 2.3 Allow deleting data from an existing system' user.

RF3. Allow the system administration to manage user roles.

3.1 Allow to assign a role to a given user.

3.2 Allow modifying the role of a given user.

RF4. Show the map given a cartography.

RF5. Allow selecting the map layers that must be visualized.

RF6. Show a smaller reference map, which indicates the portion of the map being displayed by the user.
RF7. Allow to display the graphic scale in which the map is displayed.

RF8. Allow user to traverse the map by varying its detail level.

8.1 Allow the user to zoom in the map.

8.2 Allow the user to zoom out the map.

8.3 Allow the user to display the map in its full extent.

RF9. Allow the user to display the map centered.

RF10. Allow the user to drag the map with the mouse without changing its detail level.

RF11. Allow the user to center the map in a different position by clicking the reference map.

RF12. Allow the user to select a region in the map.

12.1 Making a selection of a specific point on the map.

12.2 Making a selection of a rectangular area on the map. 12.3 Making a selection of a circumferential area on the map.

12.4 Making a selection of an area in the form of an irregular polygon on the map.

RF13. Allow the user to delete the selection.

RF14. Show the distance measurement given the selection of two points on the map.

RF15. Show the perimeter measurement of a selected area on the map.

RF16. Show the area measurement of a selected area on the map.

RF17. Show the path traveled by a vehicle from GPS device information.

RF18. Allow visualization of all data associated with a selected route.

RF19. Allow the administrator to manage the information of the fleet.

19.1 Allow adding the data of a vehicle.

19.2 Allow modifying the data values of a vehicle.

19.3 Allow deleting the data of an existing vehicle.

RF20. Allow comparison between the planned route for a vehicle and the actual traveled route during a given time interval.

RF21. Allow obtaining the optimal route between 2 points of the map for a vehicle, taking into account the distance and fuel consumption.

The requirements between RF1 and RF16 are the basis of the software and they were developed as a part of the Security, Navigation and Selection modules, they define a system able to manage users and prmissions, they also 


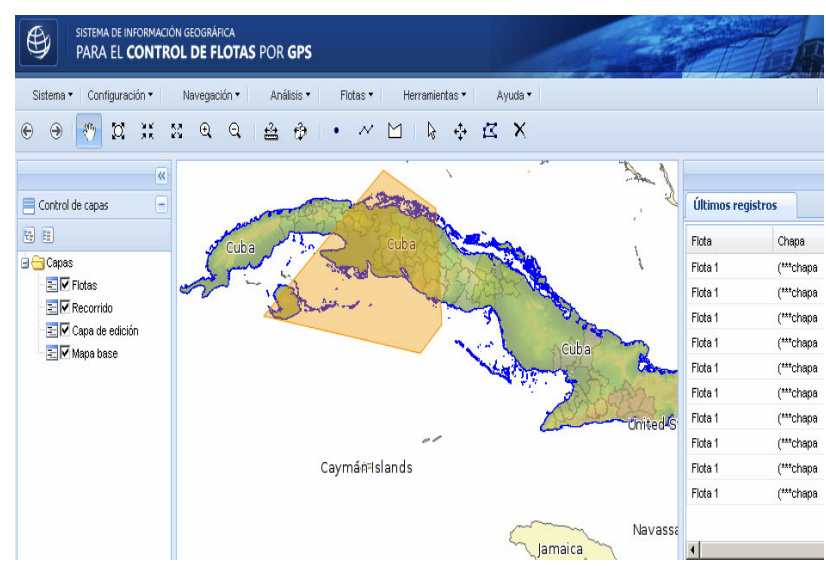

Figure 1. Performing a polygonal selection.

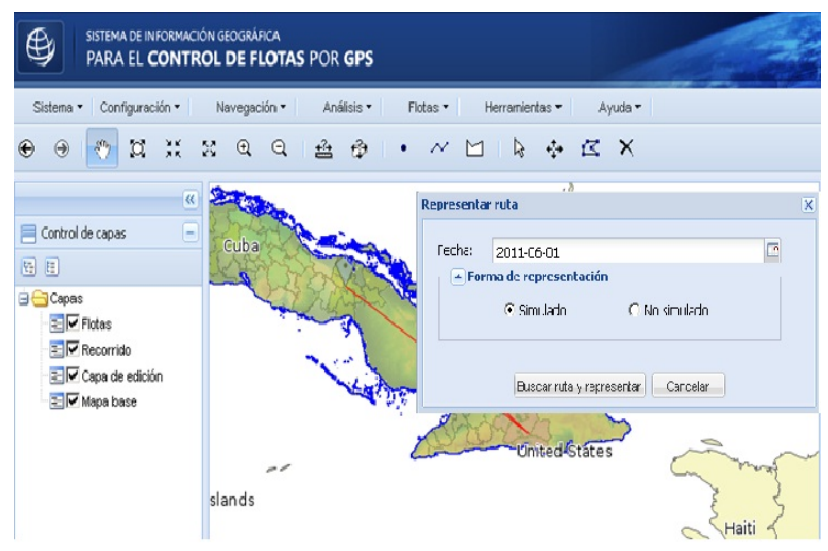

Figure 2. Showing a vehicle's route.

allow the display of a cartography that the organization using the software must select, and it is not more than the representation of the area where the organization fleet will execute its functions, also in this group are included some basic features of map analysis such as calculation of distances, areas and the selection by different ways (point, rectangle, circle and polygon) of geographic objects (vehicles, routes, checkpoints) represented in the map as you may see on Fig. 1.

The functional requirements between RF17 and RF21 are those that are part of Route Analysis module, they solve the main problems faced by a controller when monitoring a fleet, with the development of this set of requirements efficient management of the information associated to the vehicles and their routes is achieved, also a set of analysis activities regarding the behavior of the fleet in a given time interval and a set of route planning activities regarding to the routes followed by the fleet vehicles are automated as you may see on Fig. 2.

\section{CONCLUSIONS}

- The proposed system allows remote monitoring of vehicle fleets and it constitutes an useful tool to increase the effectiveness and efficiency of the fleet management process.

- The use of SIGFlotas will help organizations to achieve a reduced time response when detecting and correcting the errors related to fleet behavior.

- The proposed system allows reducing resulting errors related to the information management process by eliminating human factor from data collection.

- Technologies, tools, languages and frameworks selected for the development of the application are $100 \%$ "Open Source" and GNU GLP licensed, in agreement, they diminish the costs associated to the fleet monitoring from the fleet by avoiding the payments for software licenses.

\section{REFERENCES}

[1] Aberdeen Group. "Improving Productivity and Profitability through Service Fleet Management". [En línea] http://www.aberdeen.com/summary/report/sector insights/5006SI-service-fleets-gps.asp.

[2] Guillaumet, A., Cellini, Andres y Coen, Fernando (2000) "Minería de Datos Espaciales".

[3] Domingo Yagüez, Ing.Agr. Julio C. y Langhi, Ruben. "Sistema de Información Geográfica (S.I.G.)”. . [En línea] http://www.inta.gov.ar/barrow/info/documentos/SIG/que es sig.ht m..

[4] Linde, José Manuel Miñano. Mapping Interactivo. “¿ Qué es un SIG?" [En línea] http://www.mappinginteractivo.com/plantillaante.asp?id articulo=133..

\section{AUTHORS}

J. D. Cano is with the University of Computer Science, Carretera de San Antonio de los Baños km $2.5 \mathrm{~s} / \mathrm{n}$, La Lisa, Habana, Cuba (e-mail: jdcano@uci.cu).

M. J. Remedios is with the University of Computer Science, Carretera de San Antonio de los Baños km 2.5 s/n, La Lisa, Habana, Cuba (e-mail: mjremedios@ uci.cu).

P. E. Palau is with the University of Computer Science, Carretera de San Antonio de los Baños km 2.5 s/n, La Lisa, Habana, Cuba (e-mail: pepalau@uci.cu).

This article is an extended and modified version of a paper presented at the International Conference on Remote Engineering \& Virtual Instrumentation (REV2012), held at University of Deusto, Bilbao, Spain, July 4-6, 2012. Manuscript received 14 September 2012. Published as resubmitted by the authors 15 November 2012 . 